\title{
Hablar el idioma de la libertad. Sobre la actualidad hermenéutica del pensamiento político de Fichte ${ }^{1}$
}

Speaking the language of freedom. On the hermeneutical actuality of the political thought of Fichte ${ }^{2}$

\author{
ANDRZEJ PRZYLEBSKI \\ Adam-Mickiewicz-University Poznan (Polonia)
}

Recibido: 10-01-2014 Aceptado definitivamente: 26-03-2014

\begin{abstract}
RESUMEN
Podemos encontrar en los escritos tardíos de Fichte un giro importante desde una perspectiva individualista del Yo hacia una perspectiva comunitaria del Nosotros. Él intentó en sus Discursos a la nación alemana explicitar una relación espiritual que trabaja contra la atomatización de una sociedad dada. Elaboró para ello un interesante concepto de nación cultural. El factor constitutivo de una nación como tal es el lenguaje, y con ello: el camino del pensamiento y la experiencia de la realidad. Fue un paso adelante, no sólo hacia la famosa afirmación hegeliana acerca del progreso histórico a través de las grandes naciones, sino también hacia un giro hermenéutico en la filosofía europea. La filosofía social del Fichte tardío es una interesante mezcla de racionalismo trascendental y conciencia histórica moderna. Llevó su pensamiento a las puertas de lo que el filósofo alemán contemporáneo H. Schnadelbach ha denominado como una segunda ilustración histórico-hermenéutica.
\end{abstract}

Suplemento 19 (2014)

(C) Contrastes. Revista internacional de filosofía, pp. 249-262. ISSN: 1136-9922

Departamento de filosofía, Facultad de Filosofía y Letras, Universidad de Málaga Campus de Teatinos, E-29071 Málaga (España)

1 Título original «Sprache der Freiheit sprechen. Über die hermeneutische Aktualität des politischen Denkens von Fichte». Traducción al español de Lucas Risoto de Mesa y Alejandro Rojas Jiménez.

2 This article was written in frame of the scientific grant of NCN 2013/09/B/HS1/00282 (Hermeneutyka. Od sztuki interpretacji o teorii filozofii rozumienia). 


\author{
PALABRAS CLAVES \\ LENGUAJE, CULTURA, NACIÓN, HISTORIA HERMENÉUTICA, \\ COMUNIDAD, ATOMIZACIÓN
}

\begin{abstract}
We can find in the late writings of Fichte an important turn from an individualistic perspective of I to the community perspective of We. He tried in his Reden an die deutsche Nation to explicate a spiritual relationship that works against the atomization of a given society. He elaborated thus an interesting concept of cultural nation. The constitutive factor of such a nation is language, and with it: the ways of thinking and of experiencing the reality. It was a step ahead not only towards the famous Hegel's claim about the historical progress through the great, leading nations, but also towards a hermeneutical turn in the European philosophy. Fichte's late social philosophy is an interesting mixture of transcendental rationalism and modern historical consciousness. He situated his thought on the threshold to something the German contemporary philosopher H. Schnadelbach called a second, historicalhermeneutical Enlightenment.
\end{abstract}

\title{
KEY WORDS \\ LANGUAGE, CULTURE, NATION, HISTORY, HERMENEUTICS, COMMUNITY, ATOMIZATION
}

\section{DE CÓMO DEBE SER ESTABLECIDA ESTA ACTUALIDAD}

EL DISCURSO SOBRE LA ACTUALIDAD DE UNA TEORÍA elaborada en el idealismo alemán es siempre perspectivista, siempre se encuentra referido a una posición comprometida, es decir a una corriente filosófica en función de la cual alguien valora lo que es actual.

Esta corriente de la filosofía es para mí la filosofía hermenéutica, un modo de reflexionar y pensar que es consecuencia directa del desarrollo de la filosofía continental de la vida (Dilthey, Simmel y otros) y de la fenomenología (Husserl, Scheler, Ingarden), pasando por la discusión (especialmente clara en Ricoeur) con la que la tradición anglosajona de la filosofía analítica (el caso del linguistic turn que se observa en ella) la enriquece, e incluso la transforma.

Hay que agradecer el comienzo de la filosofía hermenéutica a Wilhelm Dilthey. Como consecuencia de su investigación metodológica y epistemológica en el campo de las ciencias humanas se le fue haciendo cada vez más claro que comprender es algo más que un método de investigación de este grupo de ciencias. Su muerte repentina en 1911 no le permitió desarrollar en su totalidad las consecuencias de este descubrimiento. ${ }^{3}$ Es por ello que el mérito de la fundación de la filosofía hermenéutica recae sobre alguien que estudió en profundidad los

3 Los enfoques de esta inversión se ven muy claramente en su obra póstuma Der Aufbau der geschichtlichen Welt in den Geisteswissenschaften sehr deutlich zu sehen. 
textos de Dilthey (aunque rara vez la admitiera). Se trata, como se puede suponer, de Martin Heidegger. Tanto en su temprana Hermenéutica de la facticidad, ${ }^{4}$ como en Ser y tiempo, su obra más madura, el comprender es concebido como el verdadero modo de ser del hombre. Se reveló la completa condición natural [voraussetzungsvolle Nature] de todo comprender, a saber como la famosa preestructura del comprender [Vorstruktur des Verstehens] que se compone de tres elementos: haber previo, manera previa de ver y la manera de entender previa.

Debido a la fama de este libro puedo ahorrarme los sucesivos detalles sobre el rol de la comprensión en el contexto del encontrarse, el habla y los demás así llamados existenciales.

Esta obra creó la ruptura. Por desgracia, el propio Heidegger apenas siguió este camino. Su posterior «pensar del ser [Seyn]» que debía reemplazar la filosofía entendida como esencialmente metafísica, no se puede considerar como una continuación de la filosofía hermenéutica, incluso aunque su nueva filosofía del lenguaje (pensemos tan solo en su célebre sentencia «el lenguaje es la casa del ser») constituya una cierta aproximación a la filosofía hermenéutica. ${ }^{5}$

Hans-Georg Gadamer recorrió otro camino. Desarrolló la hermenéutica heideggeriana de la facticidad en una dirección que él mismo denominó, desde la modestia, «hermenéutica filosófica», pero que en el fondo se trata de una nueva posición que podría denominarse tranquilamente «filosofía hermenéutica». Sus fundamentos dan forma a Verdad y método y a los otros nueve tomos de sus obras completas.

Paul Ricoeur, el tercer clásico de la filosofía hermenéutica, completa y corrige los aspectos separados de la filosofía hermenéutica y abre esta posición a una discusión con otras corrientes de la filosofía contemporánea, sobre todo la filosofía analítica, al deconstructivismo y a la teoría de la cultura de la Escuela de Frankfurt (Habermas/Apel). El mérito de Ricoeur está también en haber adquirido los logros de la filosofía americana y en haber elaborado el lenguaje de manera que se hiciera posible el diálogo productivo entre estas dos grandes tradiciones.

A la pregunta de cómo se distingue la filosofía hermenéutica respecto de otras corrientes de pensamiento de la filosofía actual, se podría -formulada en pocas palabras- dar la siguiente respuesta: la filosofía hermenéutica tematiza, de un modo análogo a como lo hace la fenomenología, el mundo humano de la propia experiencia, pero lo considera como lo hace una filosofía de la vida; no

4 Se trata de las lecciones del año 1919-1923, que bajo este título son publicadas tras la muerte de Heidegger. Significan la ruptura con respecto a los neokantianos, y un anuncio de su gran obra, que aparece en el 27.

5 Esto digo yo deliberadamente en contra del veredicto del discípulo italiano de Gadamer, G. Vattimo, quien observa en la filosofía tardía de Heidegger la continuación de la filosofía hermenéutica. Me falta en esta fase los hitos principales del pensamiento hermenéutico, como por ejemplo los papeles de la comunicación y la tradición. 
procede del conocimiento (no es un fenómeno de la conciencia), sino que procede de algo vital (de la vida práctica), es decir, de su anclaje en una situación de cooperación y comunicación entre hombres, que podríamos formular con la expresión de procesos de comunicación sociales. Comprender juega en una filosofía semejante (y con él también la interpretación: Auslegung, Deutung, Interpretation) el rol central. Por otra parte, esta filosofía pone de manifiesto que el lenguaje es el elemento en el que la comprensión y el comprender se realizan. Saber no tiene aquí el sentido de un saber puro, sino siempre de un saber referido a la vida: que sirve en última instancia-incluso cuando no es de un modo directo- a la vida, a la orientación vital que siempre se encuentra contextualizada. ${ }^{6}$

Estos son los ejes sobre los que gira la filosofía hermenéutica. Esta filosofía tiene su lado teórico y su lado práctico. Sin embargo, no se distinguen uno respecto del otro de un modo tan riguroso como ocurre por ejemplo en el caso de Kant. ${ }^{7}$ El desarrollo y la estructura de la filosofía hermenéutica lo he expuesto de un modo más detallado en mi libro sobre los cambios hermenéuticos de la filosofía. ${ }^{8}$ Las indicaciones antes mencionadas deben bastar para que no perdamos de vista el tema principal de esta exposición. A saber, la actualidad de la filosofía política de Fichte. Ahora sabemos ya que es una actualidad referida al pensamiento político en la filosofía hermenéutica.

\section{LO QUE SIGNIFICA SER ACTUAL}

Después de una investigación de muchos años sobre la filosofía clásica alemana, he llegado a la opinión de que su actualidad, es decir su aporte más vivo (no solamente histórico) a la discusión presente, se debe buscar menos en el ámbito de lo teórico que en el campo de la filosofía práctica. Para ir directamente al quid de la cuestión: no creo que la filosofía teórica de Kant (su crítica de la razón pura) describa correctamente el proceso de conocimiento en el área del conocimiento (tanto cotidiano como científico) de la naturaleza. La historización del a priori muestra que aunque las mediaciones/condiciones del conocimiento parecen evidentes a causa de un saber previo, este saber previo no tiene necesariamente que adoptar la forma de los factores «apriori» del conocimiento. Este saber previo no se tiene que tomar necesariamente como la forma de los factores kantianos apriorísticos del conocimiento (formas de la experiencia, categorías del entendimiento e ideas de la razón). Lo mismo vale según lo con-

6 Aquí está el mayor mérito del filósofo polaco, bastante desconocido, Ludwik Fleck, en lo que respecta a la comprensión hermenéutica de la incomprendida naturaleza del conocimiento. En referencia a las obras completas de Gadamer encontramos la misma indicación. Cfr., A. Przylebski, Hermeneutische Philosophie als Wiomenologische Manuskripte Vol. 12.

7 Suficiente para pensar la libertad-causalidad-esquizofrenia.

8 Cfr., A. Przylebski, Hermeneutyczny zwrot filozofii, Poznan University Press, 2005. 
cibo, y quizás incluso de un modo más contundente, para la teoría del conocimiento de Fichte y Hegel, aunque ambas concepciones ahora como antes encuentra partidarios y defensores.

En el campo de la ética y de la política se aplica otra cosa de lo que tradicionalmente se ha tratado con el título de «filosofía práctica». Las ideas de Kant y de Hegel pueden estar también en este campo llenas de vida y ser inspiradoras. $\mathrm{Y}$ en mi opinión también el pensamiento de Fichte, aunque esta actualidad es completamente reciente y un poco específica.

Los conceptos políticos de Fichte me resultan interesantes desde esta perspectiva. ¿Qué significa esto concretamente? Concretamente significa que no me interesa una reconstrucción histórico-filosófica precisa y detallada de la opinión de Fichte sobre la relación de la cultura nacional con el Estado y con el idioma o de la constelación de conceptos del cosmopolitismo-patriotismo. Para mí se trata de hacer una lectura actualizada de su escrito quizá más popular (los Discursos a la nación alemana) y desde esta perspectiva investigar el significado de nación en el marco de su pensamiento más maduro. El significado de las naciones, debería decirse en realidad, a saber para la historia universal y el orden mundial. Durante mi investigación se me han revelado interesantes perspectivas que en el interior de esta problemática conciernen sobre todo al rol del lenguaje y no solamente a la formación de la identidad nacional. El lenguaje o el carácter lingüístico del comprender, es decir, de la razón, desempeña un papel clave, como se sabe, en la filosofía hermenéutica, al menos en su variante post-gadameriana. De ahí mi justificado interés, en ver cómo el maduro y experimentado Fichte observó y distinguió esta cuestión.

Como también se sabe, los Discursos sobre la nación alemana pertenecen a la última fase de desarrollo de su filosofía, la fase en la que se comprende la importancia del colectivo en la constitución de cada individuo, y conforme a ello la libertad no se entiende desde un punto de vista individualista, sino más bien desde una perspectiva social. Este cambio del punto de vista se manifiesta aún más claramente en el Geschlossenen Handelsstaat, que fue concebido y publicado en el año 1800, es decir aproximadamente ocho años antes de los Discursos, esto es con anterioridad a la derrota de Prusia en la guerra contra Napoleón. Esta derrota, o mejor dicho, la situación espiritual que se deriva de la derrota, juega por supuesto un rol decisivo en los Discursos: Fichte quería prestar su colaboración al despertar espiritual de su derrotada nación. Sin embargo, esto solamente explica porqué se había decidido a pronunciar sus Discursos. El contenido de los discursos determinó su filosofía liberal de la acción [seine freiheitliche Philosophie der Tat]; por supuesto en su versión de aquel entonces. Y esta versión estaba determinada por otra parte por la ampliación del horizonte que su filosofía trascendental experimenta en el umbral del siglo dieciocho; una ampliación que lo conduce a desarrollar una filosofía de la historia. 
Los Discursos fueron una continuación de su conferencia sobre la filosofía de la historia que se había celebrado tres años antes (1804/05), también en Berlín. Esto ha sido reconocido explícitamente por él; en el índice de los Discursos se refiere a esta conferencia sobre los Rasgos esenciales de la época presente. La diferencia principal de ésta es que en los Discursos se defiende la transición del presente a una fase de desarrollo histórico que en los Rasgos esenciales se promete como una posibilidad futura. Repasemos: Fichte dice en su conferencia que la historia del género humano se puede dividir en cinco estadios. Que son: 1. la época de la razón instintiva, es el estadio de la inocencia del género humano; 2. la época de la Autoridad exterior a la fuerza, que no convence mediante razones: el estadio del ascenso al pecado; 3 . la época de la emancipación de toda autoridad externa, el poder de la desnuda experiencia conceptual, estadio de pecaminosidad completado; 4. el tiempo del retorno a la razón libre e interior, donde la verdad se ama y se reconoce como lo más elevado: el estadio del ascenso a la justificación, 5. la época de la realización de la razón libre e interior a todos los aspectos de la vida exterior, donde la humanidad se construye a sí misma tomando como modelo la razón: el estadio de la total justificación y santificación.

Yo no quiero realizar aquí ningún juicio definitivo sobre la legitimidad, la profundidad y el alcance de esta filosofía de la historia. Se ve, por un lado, que dicha filosofía tiene en cuenta una evolución del género humano que va desde el hombre primitivo (esto es, evolutivamente) hasta el hombre de la razón del futuro, y así, teniendo a la vista todo el anterior desarrollo de la humanización, arriesga un pronóstico sobre un estadio futuro. Y esto puede ser visto como una ventaja. Pero por otro lado concibe el proceso de la historia de forma simple y esquemática, sin tener en cuenta por ejemplo las diferentes vías de desarrollo de las distintas civilizaciones. Se debe por ello considerar, creo yo, como un tosco esbozo filosófico fundado en cierto ideal del ser humano.

Lo que otorga a los Discursos su imborrable sitio en la filosofía, y no sólo en la filosofía de la historia, es a mi juicio la consideración del rol de las naciones en el proceso de la historia. Es llamativo al mismo tiempo que Fichte no relacione esto sólo con la época post-ilustrada, con el recién comenzado siglo diecinueve, que se considera en general como el tiempo del despertar de las naciones y la construcción de los estados nacionales. No, él lo pone también en relación con la antigua Roma, una nación que habría dado forma de un modo determinante a la historia del mundo.

En este contexto, no obstante, debe ser resaltado particularmente otra cosa: que en el pensamiento de Fichte tiene lugar una conexión que es semejante a la relación del agua con el fuego, me refiero a la combinación del cosmopolitismo (como amor a toda la humanidad) con el patriotismo (el amor a la propia nación). Llega a ser posible por la aceptación de la siguiente serie: 1. que toda filosofía auténtica sólo puede tratar del hombre como tal, es decir, tiene que ser 
pensada necesariamente de forma cosmopolita; 2 . que la humanidad de su tiempo se encuentra en la cuarta fase de desarrollo; 3 . que la transición de una fase a otra depende de una forma concreta de actividad humana, es decir, que el cambio no sucede de modo automático, sino como consecuencia de ciertas acciones; que 5. para entrar en esta cuarta fase a la nación alemana, debido a su carácter nacional, le corresponden determinadas obligaciones y tareas, y que 6. a través del amor a esta nación -en este caso su propia nación- a su éxito y prosperidad, es al mismo tiempo amor a todo el género humano. De este modo llega a ser el patriotismo al mismo tiempo cosmopolitismo.

Otra consecuencia de esta cadena de argumentaciones es que sólo el patriotismo alemán es auténtico patriotismo, siendo por el contrario el patriotismo extranjero falso y equívoco. Es un modo de pensar extraño, supongo que tendríamos que estar pensando todos. $\mathrm{Y}$ al mismo tiempo un pensamiento antihermenéutico, añadiría yo. Pues en absoluto tiene en cuenta esta cadena de argumentaciones la idea de una competición, de un diálogo de culturas (también de culturas nacionales), en el sentido de una fecundación recíproca y un enriquecimiento recíproco de la humanidad. Para una posible defensa de la posición de Fichte se podría decir que él estaba obsesionado con la idea de razón (es decir, de una humanidad gobernada científicamente, en el sentido que él daba a esa palabra) y que él en segundo lugar, tendía a encomendar la realización de esta idea sobre todo a la nación alemana (aunque no exclusivamente) a causa de los rasgos del carácter alemán antes mencionados. Los méritos de la filosofía alemana de la modernidad, de las ciencias de la naturaleza y de la cultura, y de la teología, que llevan a Fichte a esta estimación, pueden suavizarnos un poco el veredicto claro e inequívoco acerca de esta desmedida exageración. Yo, dicho sea de paso, hago notar además hasta dónde soy reacio a considerar iguales los distintos aportes de las culturas nacionales individuales a la civilización europea en total, aún cuando también rechazo la prioridad indiscutible que se le da aquí a la cultura alemana.

Se puede aceptar que Fichte se había formado la opinión por aquella época de que la historia había sufrido una aceleración: un poder extranjero, en este caso, el ejército francés bajo las órdenes de Napoleón Bonaparte había llevado, e incluso obligado, a los alemanes a abandonar el estilo de vida egoístamente organizado. ${ }^{9}$ La sociedad alemana no estaba a la altura de los tiempos: la acción de los franceses le había ayudado a agotar por completo las formas de vida y de pensamiento egoístas y a volverse consciente y establecer las exigencias de la nueva época. Fichte no concibió esto como una simple espera del cumplimiento de un destino, de algo que vendría de todas maneras sin la participación humana, como se puede comprobar en muchas partes de su tratado:

9 Incluso, en una forma rudimentaria, se puede encontrar en Fichte una anticipación de la doctrina hegeliana sobre la astucia de la razón.

Suplemento 19 (2014) de Contrastes. Revista internacional de filosofía 
«Si a pesar de todo una nación hundida de tal manera pudiera salvarse a sí misma, debería hacerse usando algo antes nunca usado, mediante la creación de un orden de las cosas completamente nuevo. Por lo tanto, veamos [se dirige a los oyentes] cuál fue el motivo en el anterior orden de las cosas de que dicho orden tuviera un final necesario para que nosotros encontremos el nuevo elemento a incluir en la época que se oponga a los motivos del hundimiento anterior, para que con ello la nación hundida pueda enderezarse y comenzar una nueva vida.» ${ }^{10}$

El medio principal para la renovación espiritual de la nación alemana, aquel que no sólo garantiza a esta nación la primacía de su tarea histórica, sino ante todo asegurar la simple supervivencia o subsistencia como nación, que consiste - como saben- en una reforma fundamental de la formación: la introducción concebida de una nueva nacionalización que Fichte, partiendo de las ideas de Pestalozzi, bosqueja en los Discurso. Dejemos, sin embargo, esto de lado. Es notable, desde la perspectiva de una filosofía hermenéutica de la política, su reflexión acerca de la relación de la nación para con el Estado. Domina, me parece, la opinión de que el surgimiento de un Estado confiere a un pueblo (una comunidad formada en un idioma y con un mismo destino) la dignidad de una nación, es decir que el estado debe -digamos- marcar así el desarrollo de un pueblo o de una estirpe hasta la categoría de nación. Juega el Estado en este sentido el rol decisivo: él es el auténtico sujeto de la historia.

Fichte parece ser, en referencia a esto, de otra opinión. El Estado no tiene para él ningún valor elevadísimo, ningún fín en sí mismo -tal y como por ejemplo parece ser para Hegel-, sino que solamente se trata de un medio para un fin más alto: «el eterno y constante avance de la formación de lo puramente humano en esta nación.» ${ }^{11}$ Lo ideal, lo divino, no se desarrolla sólo a través del Estado, sino de las naciones, o mejor dicho: a través de las culturas nacionales. Los Estados sólo son formas favorables de organización y de defensa de las naciones, es decir, de los pueblos. ${ }^{12}$ Por otro lado, Fichte critica a aquellos que creen que la pérdida de la autonomía política de una nación puede tener lugar sin un profundo impacto en su propia cultura -es decir, su lenguaje, su literatura, etc. ${ }^{13}$

Con referencia a la erección de un Estado perfecto, escribe Fichte: «(...) El estado racional no se puede construir a partir de medidas artificiales del material disponible, sino que la nación debe ser para ello primeramente educada y preparada. Sólo aquella nación que haya tomado ante todo la resolución y tarea de crecer, a través del ejercicio real, en dirección hacia la perfección de la humani-

10 Cfr., J. G. Fichte, Reden an die deutsche Nation, en: J.G. Fichtes sämtliche Werke, hrsg. von Immanuel Hermann Fichte, Berlin 1845/1846, Bd. VI, S. 272.

11 Ibid., p. 392.

12 Sobre los distintos significados entre pueblo y nación en Fichte cfr., su Auffassung der Volks- und der Nationalerziehung.

13 Ibid., Reden, p. 452. 
dad, logrará después también aquel estado perfecto.» ${ }^{14}$ Tal Estado es una República, aún cuando tuviera un monarca a la cabeza. «La Nación alemana -escribe Fichte- es la única de entre las naciones europeas que a través de la acción ha demostrado capacidad en sus clases medias desde hace siglos para soportar una constitución republicana.» ${ }^{15}$

En realidad podría reducirse curiosamente la imagen fichteana de la historia mundial en tres épocas: la antigüedad, el egoísmo extranjero del presente que cree en el eterno retorno de lo mismo, y Alemania en tanto que la época del republicanismo, para la cual son determinantes las ideas de la perfección permanente (tanto de las personas como de las instituciones) y del avance histórico. ${ }^{16}$ Se observa conjuntamente que la posición de Fichte se funda en una crítica cultural de su presente, por el que no todo desarrollo tiene que significar avance, pues el hombre puede llegar, «aún cuando alguna vez alcance la cima, nuevamente a desviarse..17

Y esto por lo que, en su debida brevedad, a este aspecto de su filosofía política se refiere. Nos dedicaremos ahora a la perspectiva hermenéutica, quizás un aspecto aún más importante de los Discursos, es decir, su concepción del lenguaje, en su conexión totalmente secreta con el espíritu, de la nación y de la historia. Las referencias al lenguaje son en realidad escasas en los Discursos, no se podría hacer, creo, una detallada filosofía hermenéutica del lenguaje. Nada sorprendente por ello que Tilman Borsche no haya encontrado ningún lugar para Fichte en su reciente publicación Clásicos de la filosofia hermenéutica del lenguaje. No obstante, vale la pena reconstruir su pensamiento, al menos en parte, en relación con esta filosofía.

El primer vistazo a los Discursos muestra ya que aunque también Fichte intenta describir aquí y allí fenomenológicamente, ${ }^{18}$ su interés principal es la relevante dimensión política del lenguaje. Lenguaje -un lenguaje concreto habladole sirve para la determinación de una nación. Esto se ve en distintos sitios de su tratado. ${ }^{19}$ Tomemos por ejemplo la siguiente cita:

«como no cabe duda de que allí donde se encuentra un lenguaje particular, también existe una nación particular que tiene el derecho a organizar con independencia sus asuntos y gobernarse a sí mismos; del mismo modo se puede decir la inversa, que un pueblo que ha abandonado la tarea de su autogobierno es culpable de renunciar a su lenguaje y desaparecer confluyendo con los vencedores,

14 Ibid., pp. 353-354.

15 Ibid., p. 357.

16 Ibid., p. 362-364.

17 Ibid., p. 367.

18 Con «fenomenológico» quiero decir dos cosas conectadas: como un fenómeno complejo y como la búsqueda de la esencia del lenguaje.

19 Por ejemplo en el discurso 11., el 12. y el 13. 
produciéndose con ello la unidad, la paz interior y el olvido absoluto de las circunstancias que ya no son las que eran. $»^{20}$

Como se puede percibir en la cita de arriba, no se trata sólo del apoyo de un filósofo a los separatistas (hablaríamos hoy de Cataluña, el País Vasco o Quebec en Canadá), sino además de un programa de integración para la sociedad multicultural, cuya concepción del lenguaje propicia la constatación de Fichte de que la secreción de Prusia respecto de Alemania es artificial, al contrario que su separación respecto del resto de Europa, que «se funda en la naturaleza.» ${ }^{21} \mathrm{El}$ lenguaje es concebido por Fichte de un modo anti-individualista y anticonvencionalista. Es decir, ningún individuo tiene poder sobre el lenguaje, por ejemplo en la introducción de neologismos, es una «herramienta de la sociedad humana.» ${ }^{22}$ Un Fragmento de los Discrusos sirve claramente como demostración:

«(...) puesto que el lenguaje no está sólo mediado por el libre albedrío, sino que arranca de la comprensión de la vida como una naturalidad inmediata, así ha entrado inmediatamente en, y estimulado, la vida sin romper las leyes del lenguaje evolucionado y el poder. Como las cosas presentes en inmediatez mueven a las personas, así también las palabras de un lenguaje concreto las mueven, porque el lenguaje, como también ocurre con las cosas, de ningún modo es una chapucería arbitraria..1 ${ }^{23}$

La lengua, la lingüisticidad, pertenece de este modo en Fichte a la naturaleza -se podría decir: a la esencia- del hombre. Suena muy gadameriano la siguiente cita de los discursos: «propiamente no se habla del hombre, sino de la naturaleza humana que habla en él, y se pronuncia en sus semejantes.» ${ }^{24}$ Aquellos que estén familiarizados con la formulación gadameriana «las cosas que nos hablan, a las que nosotros, los hombres, co-respondemos en nuestro lenguaje, respondemos» ${ }^{25}$ seguro que reconocerán que es lo que se quiere decir aquí. Fichte no se olvida de que el lenguaje no es sólo el lenguaje individual hablado, sino que también - en algunos casos- se enriquece a través de nuevas creaciones y nuevos significados. Para Fichte resulta claro que la sociedad no es sólo un individuo que decide si se establece o no en el lenguaje una palabra, un carácter. Esto se

20 Reden, p. 453.

21 Así se encuentra también una formulación en el diálogo sobre el patriotismo (Los patriotas) de 1807, que se deja ver de modo implícito en los Discursos.

22 Reden, p. 314.

23 Ibid., p. 318-319.

24 Ibid., p. 314.

25 Cfr., H.-G. Gadamer, «Die Natur der Sache und die Sprache der Dinge», GW, Bd. 2, p. 66ss. 
decide a través de su aceptación en un proceso de comunicación. En la cita de arriba se divisa en mi opinión la hermenéutica trasparencia de la declaración lingüística: que a través del lenguaje se muestra un mundo (y que lo pone ante nosotros), y que el diálogo nos mueve a posicionamientos, no por sí mismo, sino a través de lo que incluye e indican.

Mucho tiempo antes del giro hermenéutico de la filosofía, Fichte toma conciencia sorprendentemente de que el lenguaje no es un sistema artificial de signos elegidos convencionalmente, sino de que en él se ha ido cristalizando hasta la actualidad ajustes semánticos y reglas sintácticas de acuerdo con la experiencia real de una sociedad lingüística, y por así decir del mundo erguido. Una cita hace una referencia muy clara al respecto:

«Si quedan designadas las comunicaciones de la convivencia, que se repiten, que están bajo las mismos influjos exteriores a la herramienta del idioma, como el idioma que tiene un pueblo de gente que se perfecciona a sí misma, entonces debemos decir: el idioma de este pueblo es necesario tal y como es, y su pueblo no expresa su conocimiento sino que más bien es el conocimiento el que se expresa a sí mismo.. ${ }^{26}$

El giro antisubjetivista que se pone de manifiesto con el linguistic turn queda iluminado aquí con mucha claridad. Pero también el papel de la tradición que es esencial para la filosofía hermenéutica: el lenguaje, en toda la amplitud de su ser, que incluye el saber real sobre la cultura de un pueblo. El mundo semántico de una noción está condicionado por su vida presente y futura. Y da información sobre los conocimientos que este pueblo ha reunido a lo largo de su historia.

Las declaraciones de Fichte sobre lenguaje van a veces incluso en la dirección de una «metafísica del lenguaje» que se engancha fácilmente a las corrientes heideggerianas. Como para Heidegger, también para Fichte el lenguaje alemán, junto con el griego, es la lengua directa de la experiencia vital. Los otros lenguajes europeos que han tomado demasiados conceptos y palabras latinas, han adoptado inconscientemente la conceptualización romana, que ya no pueden entender ni comprender como tales. Para ellos se ha perdido la posibilidad de acceder a una realidad más originaria. «Debería el discurso - dice Fichte- producirse desde los valores internos del lenguaje alemán y saltar a la palestra como uno más o menos del mismo rango, uno igualmente originario que el griego». Él habla en este contexto de una wirklich erlebten Anschaunng que a través del lenguaje puede promoverse o ocultarse.

En sintonía con Heidegger, Fichte intenta a través de la traducción al alemán desencantar ciertos conceptos fundamentales, se podría decir: que los somete a una «deconstrucción». Sucede así, a modo de ejemplo, con la palabra Idea que

26 Reden, p. 315.

Suplemento 19 (2014) de Contrastes. Revista internacional de filosofía 
reproduce a «aspecto», o con otras palabras como «Humanität, Popularität, Liberalität» que son traducidos por él a un «alemán originario», al estilo de «Menschlichkeit», «Haschen nach Gunst beim großen Haufen» und «Entfernung vom Sklavensinn». ${ }^{27} \mathrm{Y}$ él cree con ello que puede esclarecer el matiz principal, que no siempre es evaluado en un sentido positivo. Pero dejemos esto a un lado.

Suena auténticamente hermenéutico que Fichte constate que la condición de un lenguaje ejerce «una influencia imposible de medir sobre el desarrollo en su conjunto de un pueblo.» ${ }^{28} \mathrm{El}$ lenguaje acompaña al individuo en la «profundidad más recóndita de su espíritu, junto con el pensar y el querer», lo limita e inspira. El lenguaje determina el ámbito de lo incomparecible, podríamos decir con Heidegger. «La vital efectividad del pensamiento llega a elevarse a través del pensamiento y significaciones de un lenguaje vivo». ${ }^{29}$ Ante la presencia de estas formulaciones no extraña que Fichte confiera a los poetas un rol similar a algo así como creadores del pueblo, tal y como hará Heidegger 150 años más tarde. Que para él sólo el lenguaje alemán llena su presente con criterios formados a partir de la vitalidad y la experiencia, eso es ya otro tema.

\section{CONCLUSIÓN}

De lo expuesto se sacan las siguientes conclusiones:

En la última fase del desarrollo de la filosofía de Fichte tiene lugar un giro desde una perspectiva más centrada en el individuo, hacia una perspectiva más centrada en la comunidad y la sociedad.

En los Discursos a la nación alemana quiso indicar la existencia de un vínculo espiritual que podría actuar contra la atomización de la sociedad. Elaboró para ello un interesante concepto de nación cultural que estaba constituido por la comunidad lingüística y, a través de ella por: una comunidad de pensamiento y de esquemas de experiencia. Y preparó con ello la tesis hegeliana acerca de la marcha de la razón histórica a través de las naciones del mundo.

Fichte sostiene la opinión de que no carece de interés la mezcla de la moderna racionalización de la realidad con la conciencia histórica moderna. Se pone en pie en el umbral de lo que Herbert Schnädelbach II denominó como ilustración histórico-hermenéutica. El paso hacia un giro hermenéutico de la filosofía, con el que se las habieron Schleiermacher, Nietzsche, y quizás también Herder y Humboldt, no lo da Fichte. No obstante distintos aspectos de sus escritos posteriores son merecedores de ser considerados desde el punto de vista de la herme-

27 Ibid., p. 323.

28 Ibid., p. 326.

29 Ibid., p. 332.

Suplemento 19 (2014) de Contrastes. Revista internacional de filosofía 
néutica filosófica. También deben aquí ser advertidas su simplificación, errores y exageraciones. ${ }^{30}$

Nos gusta, por así decir la demostración legítima, nos irrita cuando Fichte deposita tantas esperanzas en la necesaria re-modernización de Europa a través de Alemania. Sus palabras suenan a menudo como una ilustración del eslogan «en la esencia alemana llegará el mundo a recuperarse». En el tiempo de la multiculturalidad o se ríe uno de esto, o lo condena como nacionalismo. Para aquellos que quieran hacer tal cosa, les aconsejo que lo miren desde el punto de vista de la actual Unión Europea. No sólo propuso el ministro de exterior Polaco Sikorski a Alennia hace un año, en su discurso de Berlín, de un modo inequívoco que para encargarse de la dirección de Europa, «asumirla con más responsabilidad», había que mirar al pasado sin poner la vista en lo «malo». Grecia como estado hizo exactamente lo mismo, y desde luego no se trataban sólo de palabras. Con ello no se quiere decir que yo lo encuentre correcto. Todo lo contrario. Desde el punto de vista hermenéutico es peligroso: el peligro de la monopolización de la razón que no está preparada para aprender de los demás, teniendo en cuenta las soluciones de otras naciones. No es por ello lo óptimo racionalmente.

En consecuencia, el giro hermenéutico de la filosofía producido en la segunda parte del siglo veinte (Wittgestein, Peirce, Heidegger, Gadamer) consistió en poner el lenguaje -y además la importancia de la lingüisticidad de la comprensión (Gadamer), y eso quiere decir también: las condiciones lingüísticas del pensar-en el centro del análisis filosófico; y con ello también las diferencias en la imagen de la realidad que se manifiesta y constituye a través de las diferencias lingüísticas. Merece la pena también tomar las tesis y la marcha del pensamiento (también de las argumentaciones) de Fichte bajo la lupa de la perspectiva hermenéutica para examinar su actualidad. Mi modesto trabajo pretendía sólo ser el comienzo para ello.

ANDRZej PRZYLEBSKi, es catedrático de hermenéutica de la cultura en el Institute for Cultural Studies, AMU Poznan.

Líneas de investigación:

Filosofía hermenéutica, idealismo alemán, filosofía del lenguaje, filosofía social, fenomenología.

Publicaciones recientes:

Hermeneutische Philosophie als Wissenschaft. Bemerkungen im Ausgang von Gadamer und Fleck, Rostock 2011

30 Basta citar un único ejemplo de la insolencia de Fichte: «(...) tener caracter y ser alemán, es sin duda sinónimo (...)», se dice en el cap. 12 de los Discursos (en la cita de la página 446).

Suplemento 19 (2014) de Contrastes. Revista internacional de filosofía 
Dlaczego Polska jest wartościa. Wprowadzenie do hermeneutycznej filozofii polityki, Wyd. Poznańskie, Poznań 2013

Sense, Meaning and Understanding. Towards a Systematic Hermeneutical Philosophy, LIT Verlag, Berlin 2013. $169 \mathrm{~s}$.

Dirección electrónica: andrzej@przylebski.com 